\title{
The Effect of Total Tumor Volume on the Biologically Effective Dose to Tumor and Kidneys for ${ }^{177}$ Lu-Labeled PSMA Peptides
}

\author{
Nusrat J. Begum*1, Anne Thieme*2, Nina Eberhardt ${ }^{2}$, Robert Tauber ${ }^{3}$, Calogero D’ Alessandria $^{2}$, Ambros J. Beer ${ }^{1}$, \\ Gerhard Glatting ${ }^{1}$, Matthias Eiber ${ }^{\dagger 2}$, and Peter Kletting ${ }^{\dagger 1}$ \\ ${ }^{I}$ Department of Nuclear Medicine, Universität Ulm, Ulm, Germany; ${ }^{2}$ Department of Nuclear Medicine, Klinikum Rechts der Isar, \\ Technische Universität München, Munich, Germany; and ${ }^{3}$ Department of Urology, Klinikum Rechts der Isar, Technische Universität \\ München, Munich, Germany
}

The aim of this work was to simulate the effect of prostate-specific membrane antigen (PSMA)-positive total tumor volume (TTV) on the biologically effective doses (BEDs) to tumors and organs at risk in patients with metastatic castration-resistant prostate cancer who are undergoing ${ }^{177} \mathrm{Lu}-\mathrm{PSMA}$ radioligand therapy. Methods: A physiologically based pharmacokinetic model was fitted to the data of 13 patients treated with ${ }^{177} \mathrm{Lu}-\mathrm{PSMA}$ I\&T (a PSMA inhibitor for imaging and therapy). The tumor, kidney, and salivary gland BEDs were simulated for TTVs of $0.1-10 \mathrm{~L}$. The activity and peptide amounts leading to an optimal tumor-to-kidneys BED ratio were also investigated. Results: When the TTV was increased from 0.3 to $3 \mathrm{~L}$, the simulated BEDs to tumors, kidneys, parotid glands, and submandibular glands decreased from $22 \pm 15$ to $11.0 \pm 6.0 \mathrm{~Gy}_{1.49}, 6.5 \pm$ 2.3 to $3.7 \pm 1.4 \mathrm{~Gy}_{2.5}, 11.0 \pm 2.7$ to $6.4 \pm 1.9 \mathrm{~Gy}_{4.5}$, and $10.9 \pm 2.7$ to $6.3 \pm 1.9 \mathrm{~Gy}_{4.5}$, respectively (where the subscripts denote that an $\alpha / \beta$ of $1.49,2.5$, or 4.5 Gy was used to calculate the BED). The BED to the red marrow increased from $0.17 \pm 0.05$ to $0.32 \pm 0.11 \mathrm{~Gy}_{15}$. For patients with a TTV of more than $0.3 \mathrm{~L}$, the optimal amount of peptide was $273 \pm 136 \mathrm{nmol}$ and the optimal activity was $10.4 \pm 4.4$ GBq. Conclusion: This simulation study suggests that in patients with large PSMA-positive tumor volumes, higher activities and peptide amounts can be safely administered to maximize tumor BEDs without exceeding the tolerable BED to the organs at risk.

Key Words: prostate-specific membrane antigen (PSMA); biologically effective dose (BED); total tumor volume (TTV); physiologically based pharmacokinetic (PBPK) modeling

J Nucl Med 2018; 59:929-933

DOI: 10.2967/jnumed.117.203505

\section{$\mathbf{R}$} adioligand therapy (RLT) using radiolabeled prostate-specific membrane antigen (PSMA)-specific peptides has been proven to be a safe, effective, and thus promising method for the treatment of metastatic castration-resistant prostate cancer (1-4). A oneactivity-fits-all approach is widely used. However, RLT might be

Received Oct. 10, 2017; revision accepted Jan. 20, 2018

For correspondence or reprints contact: Peter Kletting, Klinik für Nuklearmedizin, Universität Ulm, D-89081 Ulm, Germany.

E-mail: peter.kletting@uniklinik-ulm.de

${ }^{*}$ Contributed equally to this work.

tContributed equally to this work.

Published online Feb. 1, 2018.

COPYRIGHT (c) 2018 by the Society of Nuclear Medicine and Molecular Imaging. improved by considering the high interpatient pharmacokinetic variability reported in several dosimetry studies (5-8). In RLT with somatostatin receptor type 2 -specific peptides, it has been shown that tumor burden, body size, and renal function significantly affect the activity concentration in organs at risk (OARs) and tumor tissue (9). However, so far, in patients with metastatic castration-resistant prostate cancer, a standard activity and peptide amount is usually injected, although the tumor burden varies considerably (from milliliters to several liters). In a recent study, it was shown that large tumor loads lead to a considerably lower $\mathrm{SUV}_{\text {mean }}$ in dose-limiting organs (10). Therefore, the biologically effective doses (BEDs) to normal tissues and tumor tissue might also vary considerably (9). For example, large tumor burdens most presumably lead to a less effective treatment when a standard activity and amount of peptide are used (9). Physiologically based pharmacokinetic (PBPK) models are most suitable to systematically and quantitatively investigate important pharmacokinetic parameters, because they take into account the individual patient physiology of the OARs and the pathophysiology of the tumor $(8,11,12)$.

The aims of this study were to quantitatively investigate, for ${ }^{177}$ Lu-labeled PSMA-specific peptides, the effects of different total tumor volumes (TTVs) and varying activities and peptide amounts on the BEDs to tumors and OARs. Therefore, the parameters of a recently developed PBPK model (8) were simultaneously fitted to the PET/CT and planar imaging data of 13 patients treated with RLT with ${ }^{177} \mathrm{Lu}-\mathrm{PSMA}$ I\&T (1). On the basis of the estimated parameters, simulations were conducted for a range of tumor volumes $(0.1-10 \mathrm{~L})$ and different peptide amounts and activities.

\section{MATERIALS AND METHODS}

\section{Patients and Therapeutic Measurements}

The data of 13 patients with metastatic castration-resistant prostate cancer treated with ${ }^{177}$ Lu-PSMA I\&T RLT were included (Table 1 and Supplemental Table B1; supplemental materials are available at http:// jnm.snmjournals.org). These patients were also part of a recently published study (1). The inclusion criteria were at least 3 whole-body planar scintigraphs, including 1 late scintigraph (6-8 d after injection) (Supplemental Table B2). Only the first cycle of RLT was used. The patients received an average $( \pm \mathrm{SD})$ of $91 \pm 5.0 \mathrm{nmol}$ of ${ }^{177} \mathrm{Lu}$-PSMA I\&T labeled with $7.3 \pm 0.34 \mathrm{GBq}$ of ${ }^{177} \mathrm{Lu}(1)$. Amino acids were coinfused as applied in peptide receptor radionuclide therapy. Quantification 
TABLE 1

Patient Data

\begin{tabular}{|c|c|c|c|c|c|c|c|c|c|}
\hline \multirow[b]{2}{*}{ Patient no. } & \multirow[b]{2}{*}{ Age (y) } & \multirow[b]{2}{*}{$\mathrm{BSA}\left(\mathrm{m}^{2}\right)$} & \multirow[b]{2}{*}{ PSA (ng/L) } & \multicolumn{6}{|c|}{ Measured volumes (mL) } \\
\hline & & & & Kidneys & SM glands & $\begin{array}{l}\text { Parotid } \\
\text { glands }\end{array}$ & Tumor lesion 1 & Tumor lesion 2 & Tumor REST \\
\hline 1 & 66 & 2.0 & 513 & 372 & 17 & 39 & 9.4 & 19 & 201 \\
\hline 2 & 75 & 1.8 & 49 & 273 & 17 & 46 & 2.1 & 4.2 & 130 \\
\hline 3 & 72 & 2.0 & 31 & 304 & 13 & 43 & 14 & 52 & 156 \\
\hline 4 & 66 & 1.9 & 97 & 282 & 14 & 26 & 14 & 31 & 370 \\
\hline 5 & 74 & 1.9 & 47 & 281 & 19 & 44 & 26 & 17 & 686 \\
\hline 6 & 58 & 2.2 & 2,905 & 400 & 13 & 36 & 81 & 92 & 5,620 \\
\hline 7 & 64 & 1.9 & 0.23 & 278 & 16 & 21 & 48 & 37 & 17 \\
\hline 8 & 73 & 2.0 & 1,193 & 233 & 18 & 45 & 5.7 & 19 & 429 \\
\hline 9 & 77 & 1.8 & 2,547 & 256 & 12 & 41 & 15 & 8.7 & 1,481 \\
\hline 10 & 63 & 2.0 & 2,471 & 392 & 13 & 40 & 1.9 & 5.3 & 252 \\
\hline 11 & 73 & 1.8 & 7 & 305 & 21 & 39 & 0.33 & 1.2 & 10 \\
\hline 12 & 74 & 2.0 & 161 & 297 & 13 & 59 & 9.9 & 8.7 & 217 \\
\hline 13 & 74 & 2.1 & 133 & 348 & 13 & 48 & 6.1 & 2.0 & 17 \\
\hline Mean & 70 & 2.0 & 781 & 309 & 15.2 & 40.6 & 18 & 23 & 737 \\
\hline SD & 5.8 & 0.1 & 1,112 & 53 & 3.0 & 9.7 & 23 & 26 & 1,519 \\
\hline
\end{tabular}

$\mathrm{BSA}=$ body surface area; PSA = prostate-specific antigen; $\mathrm{SM}=$ submandibular.

was performed as previously described (1). The time-activity data of the kidneys, 2 tumor lesions showing no significant overlap with other PSMAligand-positive tissues, and the total body were used, and the activities in underlying tissue (muscle and adipose) were corrected within the PBPK model (Supplemental Eq. B1). The institutional review board of the Technische Universität München approved the compassionate use of ${ }^{177} \mathrm{Lu}-$ PSMA I\&T in metastatic castration-resistant prostate cancer patients who had no other therapeutic options. All subjects signed a written informed consent form.

\section{Pretherapy Measurements}

The ${ }^{68} \mathrm{Ga}$-PSMA HBED-CC $(1.6 \pm 0.3$ nmol labeled with $115 \pm$ $16 \mathrm{MBq}) \mathrm{PET} / \mathrm{CT}$ images before therapy (1-9 wk) were used to determine the volumes for tumors, kidneys, salivary glands, and lacrimal glands (1) with SyngoVia (Siemens Healthcare). TrueD (Siemens Healthcare) was used to estimate the activity concentration. Correction for background activity in the tumor lesion region of interest was performed (Supplemental Eq. B2). To estimate the volume of a single tumor lesion, the volume of interest was defined with an isocontour of $20 \%-50 \%$ of $\mathrm{SUV}_{\max }$, with adjustment of the volume of interest to the anatomic configuration of the lesion based on the CT scan (1). The cumulated activity and volume of all tumor lesions other than lesions 1 and 2 were obtained by adding all lesions slice by slice using isocontours of $15 \%-20 \%$. This cumulated volume, which consisted of hundreds of lesions, is given the term tumor REST here. For this tumor REST mass, CT was not used. In PBPK models, measured data can be linked to several compartments (Supplemental Eqs. B1-B4). The $\mathrm{PET} / \mathrm{CT}$ data of the kidney and the 2 tumor lesions were corrected for background using 2 different volumes of interest (Supplemental Eqs. B2 and B4). The PET/CT data of the tumor REST were assigned to the pertaining compartments, with a correction factor considering potential over- or underestimation (Supplemental Eq. B3; Supplemental Table C1).
Therefore, to correct for the overestimation or underestimation of the tumor volume, a correction factor was introduced and estimated in the fitting process (Supplemental Eq. B3).

For the kidneys, the activity concentration was derived using the postprocessing software Syngo TrueD (Siemens Healthcare) with 15\% or $20 \%$ of $\mathrm{SUV}_{\text {max }}$ and correction of background activity (Supplemental Eq. B4).

\section{PBPK Modeling}

To investigate the influence of the TTV on the BED, the application of PBPK modeling is required, as the tumor volume and all parameters that determine the biokinetics are explicitly modeled. A whole-body PBPK model for PSMA-specific ligands was recently developed (8). To account for tumor growth between the PET/CT imaging and the therapy, the PBPK model was modified to include exponential tumor growth (Supplemental Table A1). The assignment of data to the specific compartments was also improved (part B of the supplemental materials). The model includes all physiologically and physically relevant mechanisms such as blood flow, PSMA-specific binding, internalization and release of ${ }^{177} \mathrm{Lu}$ from the cells, excretion, and physical decay. The model and parameters are described in detail in part A of the supplemental materials.

The simulation analysis and modeling software (SAAM II and Popkinetics, version 2.2; The Epsilon Group) was used for fitting (8). For the 2 investigated tumor lesions, the receptor density, $R_{\mathrm{TU}, 0}$, and the blood flow density, $f_{\mathrm{TU}}$, were estimated for each patient. The cell release rates of all tumor lesions, $\lambda_{\mathrm{TU}, \text { release, }}$, were assumed to be equal within one patient. For the kidneys, the blood flow density, $f_{K}$; receptor

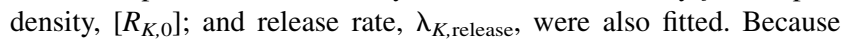
differences in salivary and lacrimal gland uptake do not considerably alter the tumor and kidney biokinetics, and planar imaging is inaccurate for these small organs, we assumed the literature values for the receptor densities and flows (8). The fitting was conducted in a Bayesian framework. Goodness of fit was evaluated using visual inspection, 
the SE of the fitted parameters, the correlation matrix, and the coefficient of determination (13).

\section{BEDs}

The BEDs to each lesion were calculated (Supplemental Eqs. A14A18) on the basis of the determined parameters and the radiobiologic parameters (for tumors, $\alpha / \beta=1.49 \mathrm{~Gy}$ (14) and a repair half-life $\mu$ of $1.9 \mathrm{~h}(14)$ ) from radiotherapy and brachytherapy. For calculation of the absorbed dose to the kidneys, salivary glands, and tumors, only the self-dose was considered. Determination of the $\mathrm{S}$ values for each tumor lesion and the salivary glands was based on the data of OLINDA/EXM for ${ }^{177} \mathrm{Lu}$ for spheres (Supplemental Table A2). The BED model for red marrow was implemented according to Hindorf et al. (15) and calculated as described in part $\mathrm{C}$ of the supplemental materials.

\section{Simulations}

For the simulations and BED calculations, MATLAB/Simulink, version 2015b (The MathWorks, Inc.), was used. On the basis of the fitted parameters, the pharmacokinetics were simulated for, first, a TTV (i.e., the volume of the tumor REST plus the 2 tumor lesions) of $0.1,0.3,1,3$, and $10 \mathrm{~L}$ and, second, varying combinations of peptide amount $\left(2-2^{10} \mathrm{nmol}\right)$ and activity leading to a kidney dose of $5 \mathrm{~Gy}_{2.5}$ (where the subscript denotes that an $\alpha / \beta$ of 2.5 Gy was used to calculate the BED). The limit of $5 \mathrm{~Gy}_{2.5}$ was chosen because it is a commonly assumed tolerable dose to the kidneys allowing multiple cycles-for example, 8 when assuming a maximal cumulative dose of $40 \mathrm{~Gy}_{2.5}$ and no additional risk factors. With these simulations, the BEDs were obtained for 2 tumor lesions, the tumor REST, the salivary glands, and the kidneys. The ratio of tumor BED to kidney BED was also determined. Mean values were derived for all patients and for just patients with a TTV of $0.3-3 \mathrm{~L}$.

Dosimetry models for the salivary glands and the bone marrow (especially for highly metastasized bones and diffuse red marrow infiltration) include large uncertainties. However, to see the potential influence of the TTV (despite the known uncertainties) on the red marrow BED and salivary gland BED, the simulations were also conducted for these organs.

\section{RESULTS}

The goodness of fits was excellent for all patients as ascertained by visual inspection and the coefficient of determination $\left(R^{2}>\right.$ 0.9) (Supplemental Fig. C1). All elements of the correlation matrix were within -0.8 and 0.8 in all patients. The relative SD for all fitted parameters in all patients was less than $28 \%$. All estimated parameter values (Supplemental Table C2) were in a physiologically reasonable range.

The dependence of BED on TTV is shown in Figure 1 for a typical patient (patient 4$)$ with high tumor receptor densities $(\sim 80$ $\mathrm{nmol} / \mathrm{L})$. The results for all patients are provided in Supplemental Table C3A for BED and in Supplemental Table C3B for absorbed dose. The decreases in BED within a TTV of 0.3-3 L were considered most relevant, because the median of the patients' TTV was $0.22 \mathrm{~L}$; 10 of 13 patients had a TTV of more than $0.1 \mathrm{~L}$ and only one a TTV of more than $3 \mathrm{~L}$. The simulated BEDs to the tumor lesions and kidneys decreased by $58.2 \%$ and $58.4 \%$, respectively, from a TTV of $0.3 \mathrm{~L}$ to a TTV of $3 \mathrm{~L}$ (Table 2). For all patients, the changes in the tumor-to-kidney BED ratio for standard amounts of peptide and activities were maximally $12 \%$. The BEDs to the submandibular glands, parotid glands, and red marrow changed by $59 \%, 59 \%$, and $185 \%$, respectively, from a

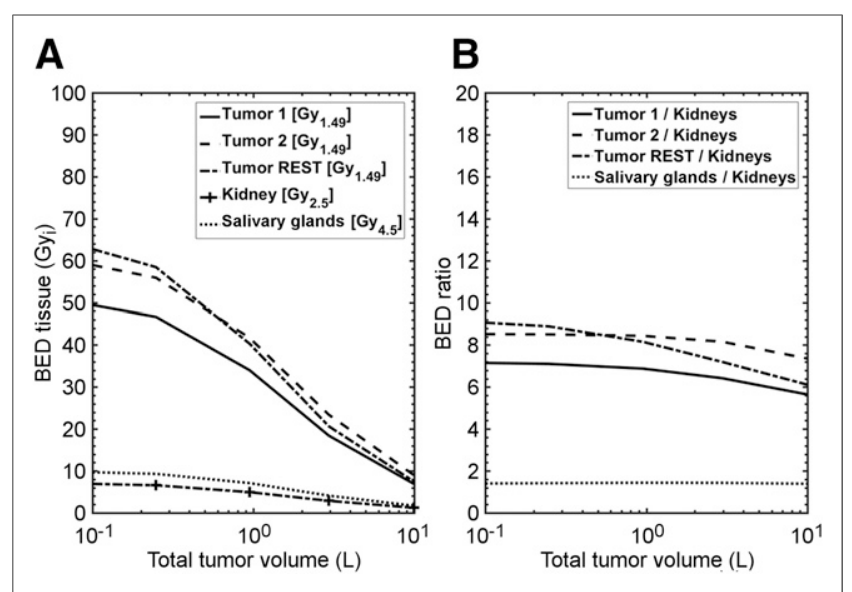

FIGURE 1. (A) Dependence of BED on TTV is shown for patient 4. (B) Tumor-to-kidney and salivary gland-to-kidney ratios with increasing TTV show that despite large absolute reduction in BEDs, ratios are only minimally influenced. Submandibular and parotid glands are depicted in single line as salivary glands because the values of the two are very similar to each other.

TTV of $0.3 \mathrm{~L}$ to a TTV of $3 \mathrm{~L}$ (Table 2). For a TTV of $10 \mathrm{~L}$, the average BED to the bone marrow increased to $0.39 \mathrm{~Gy}_{15}$.

Figure 2 shows the optimal amount of peptide and pertaining activity for patient 4 with the maximal BED to the kidneys fixed to $5 \mathrm{~Gy}_{2.5}$. The individual results for all patients are provided in Supplemental Table C4. Patients with a high tumor burden $(>0.3 \mathrm{~L})$ could be administered higher activities $(10.4 \pm 4.4 \mathrm{GBq})$ and peptide amounts $(273 \pm 136 \mathrm{nmol})$ without exceeding the tolerable BED to the kidneys.

\section{DISCUSSION}

The influence of larger tumor masses on pharmacokinetics is known as the tumor sink effect (9). This may lead to decreased activity concentrations in healthy tissue (10) but also in the tumor, with the risk of underdosing the tumor. In this work, we have quantitatively investigated the influence of TTV on the pharmacokinetics and thus on the BED to the OARs and tumors for PSMAspecific peptides. We used a recently developed PBPK model (8) that was simultaneously fitted to PET/CT and planar images to determine the pharmacokinetic parameters for each patient. The PET/CT information and the PBPK model were used to correct for nontumor activity in the tumor regions of interest on the planar images. However, SPECT/CT imaging during therapy, assuming a well-calibrated measurement system, would improve the accuracy of the individual pharmacokinetic parameters. An approximately 50-fold higher amount of peptide was used for therapeutic applications than for PET imaging. The parameters therefore describe the pharmacokinetics of smaller amounts and larger amounts, thus improving the reliability of the simulations. Within the PBPK model, the molecular weight, affinity, activity, physical decay, and peptide amount of the ${ }^{68} \mathrm{Ga}-\mathrm{PSM} A \mathrm{HBED}-\mathrm{CC}$ and ${ }^{177} \mathrm{Lu}-$ PSMA I\&T injections were considered. The fitted parameters were in a physiologically reasonable range. On the basis of these parameters (e.g., receptor densities), simulations with TTVs of between 0.1 and $10 \mathrm{~L}$ were conducted. The BED to the kidneys and tumors decreased considerably. Our results are remarkably similar to the $\mathrm{SUV}_{\text {mean }}$ decline in kidneys (55\%), submandibular glands 


\begin{tabular}{|c|c|c|c|}
\hline \multirow[b]{2}{*}{ Organ } & \multicolumn{3}{|c|}{ BED for TTV of... } \\
\hline & $0.3 \mathrm{~L}$ & $1 \mathrm{~L}$ & $3 \mathrm{~L}$ \\
\hline Tumor lesion $\left(\mathrm{Gy}_{1.49}\right)$ & $22 \pm 15$ & $18 \pm 11(84.2 \% \pm 8.8 \%)$ & $11.0 \pm 6.0(58.2 \% \pm 17.5 \%)$ \\
\hline Tumor REST (Gy $\left.{ }_{1.49}\right)$ & $26 \pm 16$ & $20 \pm 10(81 \% \pm 10 \%)$ & $11.4 \pm 4.7(53 \% \pm 19 \%)$ \\
\hline Kidneys $\left(\mathrm{Gy}_{2.5}\right)$ & $6.5 \pm 2.3$ & $5.4 \pm 1.8(84.3 \% \pm 8.4 \%)$ & $3.7 \pm 1.4(58.4 \% \pm 16.6 \%)$ \\
\hline Submandibular glands $\left(\mathrm{Gy}_{4.5}\right)$ & $10.9 \pm 2.7$ & $9.2 \pm 2.1(84.6 \% \pm 8.0 \%)$ & $6.3 \pm 1.9(59 \% \pm 16 \%)$ \\
\hline Parotid glands $\left(\mathrm{Gy}_{4.5}\right)$ & $11.0 \pm 2.7$ & $9.2 \pm 2.1(84.6 \% \pm 8.1 \%)$ & $6.4 \pm 1.9(59 \% \pm 16 \%)$ \\
\hline Red marrow (Gy $\left.y_{15}\right)$ & $0.17 \pm 0.05$ & $0.23 \pm 0.08(136 \% \pm 15 \%)$ & $0.3 \pm 0.1(185 \% \pm 29 \%)$ \\
\hline \multicolumn{4}{|c|}{ Data in parentheses are relative to $0.3 \mathrm{~L}$. } \\
\hline
\end{tabular}

(64\%), and parotid glands (57\%) between patients with a low tumor load and patients who were classified as having a "high tumor load" in a study by Gaertner et al. (10). The increase in red marrow BED with increasing tumor burden occurs because of higher total-body retention.

Garske et al., in a case report on ${ }^{177} \mathrm{Lu}$ DOTA-octreotate therapy, noted that one lesson learned was the relevance of the amount of peptide relative to the number of receptors (10). With increasing tumor load (using the same activity and peptide amount), the tumor-to-kidney BED ratio slightly decreased as the saturation effects of the kidneys were reduced with less available peptide. However, the ratio can be improved by applying higher amounts of peptide with adapted activity. For antibodies (17-19) and peptides $(8,11,20)$, it has been shown that an optimal tumor-to-OAR ratio exists for each patient. Using an algorithm developed by Kletting et al. (11), we determined, for each individual patient, the optimal activity and peptide amount for a maximal kidney BED of $5 \mathrm{~Gy}_{2.5}$ for 1 cycle. The tumor radiobiologic parameters from Fowler et al. (14) were used, although there is ongoing controversy about the actual range (21) and its applicability to RLT. Therefore, the absorbed doses and the BED for an $\alpha / \beta$ of 1.8 , reported by Dearnaley et al. (21), are also provided in Supplemental Tables C3B and C3C. The maximal deviation for the

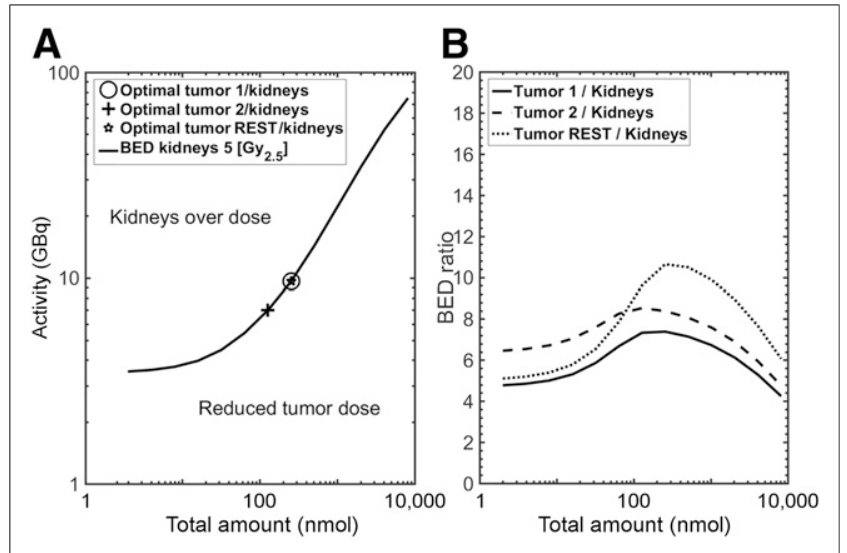

FIGURE 2. Optimal combination of peptide amount and activity for tumor lesions and tumor REST with maximal BED to kidneys of 5 $\mathrm{Gy}_{2.5}$ is shown for patient 4 (high receptor density and TTV of $0.37 \mathrm{~L}$ ). (A) All combinations of activity and peptide amount that lead to BED of $5 \mathrm{~Gy}_{2.5}$ are shown. (B) Most favorable combination is maximal graphed point. tumor BED was $5 \%$ over the range of $\alpha / \beta$ values. The results indicate that through individualized dosimetry based on our PBPK model, the BED to the tumors could be safely maximized by optimizing the tumor-to-kidney BED ratio for a given limiting BED. This holds especially true for patients with a high tumor burden. However, because the number of possible cycles could also be limited by the bone marrow BED (Supplemental Table C4) (22) or the salivary gland BED, an overall optimization approach simultaneously taking into account all OARs and treatment effects is desirable. Future studies with quantitative 3-dimensional imaging (during therapy) are required to accurately estimate the parameters for the salivary (and lacrimal) glands. In addition, improved models for red marrow dosimetry are needed to accurately estimate the red marrow dose. The Simulink model and the algorithm can easily be modified to consider more than one OAR or limiting dose.

The idea of using the diagnostic PET/CT scan for treatment planning (23) is intriguing but requires several elements: a PPBK model that considers different affinities, peptide amounts, and half-lives of the PET and therapeutic agents; population parameters with an acceptably low SD for the release rates and the receptor densities; modeling of tumor growth and potential changes in salivary gland function due to cooling or candies; and an algorithm that objectively determines the PSMA-positive tumor volume without information on the therapeutic planar total-body time-activity data. Then, future clinical management could be changed by adapting the activity and peptide amount individually before the first therapeutic cycle. Individual adaptation is also relevant for early phases of the disease with a low tumor burden, as the optimal combination of activity and peptide amount might be considerably different from that in the patient population with a high tumor burden.

\section{CONCLUSION}

This modeling and simulation study indicated that the tumor burden considerably affects the BEDs to tumors and OARs. Our data suggest that patients with a large PSMA-ligand-positive tumor mass might be safely administered higher activities and peptide amounts to maximize the tumor BED without exceeding the critical dose to the OARs.

\section{DISCLOSURE}

This work was supported by LPDP-Lembaga Pengelola Dana Pendidikan (the Indonesia Endowment Fund for Education, 
Ministry of Finance, Indonesia) (s-2512/LPDP.3/2016) and the Deutsche Forschungsgemeinschaft (the German Research Foundation) (KL2742/2-1, BE4393/1-1, GL236/11-1, and SFB824 project B11). No other potential conflict of interest relevant to this article was reported.

\section{REFERENCES}

1. Okamoto S, Thieme A, Allmann J, et al. Radiation dosimetry for Lu-177-PSMA I\&T in metastatic castration-resistant prostate cancer: absorbed dose in normal organs and tumor lesions. J Nucl Med. 2017;58:445-450.

2. Rahbar K, Ahmadzadehfar H, Kratochwil C, et al. German multicenter study investigating ${ }^{177} \mathrm{Lu}$-PSMA-617 radioligand therapy in advanced prostate cancer patients. J Nucl Med. 2017;58:85-90.

3. Baum RP, Kulkarni HR, Schuchardt C, et al. ${ }^{177}$ Lu-labeled prostate-specific membrane antigen radioligand therapy of metastatic castration-resistant prostate cancer: safety and efficacy. J Nucl Med. 2016;57:1006-1013.

4. Kratochwil C, Giesel FL, Stefanova M, et al. PSMA-targeted radionuclide therapy of metastatic castration-resistant prostate cancer with ${ }^{177} \mathrm{Lu}$-labeled PSMA617. J Nucl Med. 2016;57:1170-1176.

5. Delker A, Fendler WP, Kratochwil C, et al. Dosimetry for ${ }^{177}$ Lu-DKFZ-PSMA617: a new radiopharmaceutical for the treatment of metastatic prostate cancer. Eur J Nucl Med Mol Imaging. 2016;43:42-51.

6. Kabasakal L, AbuQbeitah M, Aygun A, et al. Pre-therapeutic dosimetry of normal organs and tissues of ${ }^{177}$ Lu-PSMA-617 prostate-specific membrane antigen (PSMA) inhibitor in patients with castration-resistant prostate cancer. Eur J Nucl Med Mol Imaging. 2015;42:1976-1983.

7. Scarpa L, Buxbaum S, Kendler D, et al. The ${ }^{68} \mathrm{Ga} /{ }^{177} \mathrm{Lu}$ theragnostic concept in PSMA targeting of castration-resistant prostate cancer: correlation of SUVmax values and absorbed dose estimates. Eur J Nucl Med Mol Imaging. 2017;44:788800 .

8. Kletting P, Schuchardt C, Kulkarni HR, et al. Investigating the effect of ligand amount and injected therapeutic activity: a simulation study for ${ }^{177} \mathrm{Lu}$-labeled PSMA-targeting peptides. PLoS One. 2016;11:e0162303.

9. Beauregard JM, Hofman MS, Kong G, Hicks RJ. The tumour sink effect on the biodistribution of ${ }^{68} \mathrm{Ga}$-DOTA-octreotate: implications for peptide receptor radionuclide therapy. Eur J Nucl Med Mol Imaging. 2012;39:50-56.
10. Gaertner FC, Halabi K, Ahmadzadehfar H, et al. Uptake of PSMA-ligands in normal tissues is dependent on tumor load in patients with prostate cancer. Oncotarget. 2017;8:55094-55103.

11. Kletting P, Kull T, Maass C, et al. Optimized peptide amount and activity for ${ }^{90}$ Y-labeled DOTATATE therapy. $J$ Nucl Med. 2016;57:503-508.

12. Nestorov I. Whole-body physiologically based pharmacokinetic models. Expert Opin Drug Metab Toxicol. 2007;3:235-249.

13. Kletting P, Schimmel S, Kestler HA, et al. Molecular radiotherapy: the NUKFIT software for calculating the time-integrated activity coefficient. Med Phys. 2013; 40:102504.

14. Fowler J, Chappell R, Ritter M. Is alpha/beta for prostate tumors really low? Int J Radiat Oncol Biol Phys. 2001;50:1021-1031.

15. Hindorf C, Glatting G, Chiesa C, Lindén O, Flux G. EANM Dosimetry Committee guidelines for bone marrow and whole-body dosimetry. Eur J Nucl Med Mol Imaging. 2010;37:1238-1250.

16. Garske U, Sandström M, Johansson S, et al. Lessons on tumour response: imaging during therapy with ${ }^{177} \mathrm{Lu}$-DOTA-octreotate: a case report on a patient with a large volume of poorly differentiated neuroendocrine carcinoma. Theranostics. 2012;2:459-471.

17. Kletting P, Bunjes D, Reske SN, Glatting G. Improving anti-CD45 antibody radioimmunotherapy using a physiologically based pharmacokinetic model. J Nucl Med. 2009;50:296-302.

18. Kletting P, Kull T, Bunjes D, Luster M, Reske SN, Glatting G. Optimal preloading in radioimmunotherapy with anti-cD45 antibody. Med Phys. 2011;38:2572-2578.

19. Pandit-Taskar N, O’Donoghue JA, Divgi CR, et al. Indium 111-labeled J591 antiPSMA antibody for vascular targeted imaging in progressive solid tumors. EJNMMI Res. 2015;5:28.

20. Kletting P, Muller B, Erentok B, et al. Differences in predicted and actually absorbed doses in peptide receptor radionuclide therapy. Med Phys. 2012;39:5708-5717.

21. Dearnaley D, Syndikus I, Mossop H, et al. Conventional versus hypofractionated high-dose intensity-modulated radiotherapy for prostate cancer: 5-year outcomes of the randomised, non-inferiority, phase 3 CHHiP trial. Lancet Oncol. 2016;17: 1047-1060.

22. Rathke H, Giesel FL, Flechsig P, et al. Repeated Lu-177-PSMA-617 radioligand therapy using treatment activities up to $9.3 \mathrm{GBq}$. J Nucl Med. August 10, 2017 [Epub ahead of print].

23. Hardiansyah D, Maass C, Attarwala AA, et al. The role of patient-based treatment planning in peptide receptor radionuclide therapy. Eur J Nucl Med Mol Imaging. 2016;43:871-880. 